\title{
Targeted next generation sequencing reveals a novel intragenic deletion of the $L A M A 2$ gene in a patient with congenital muscular dystrophy
}

\author{
YUN YANG $^{1,2^{*}}$, BING MAO $^{3,4^{*}}$, LIXIA WANG $^{5}$, LIANGWEI MAO ${ }^{1}$, AIFEN ZHOU $^{2}$, JIANGXIA CAO $^{2}$, \\ JIASHENG HU ${ }^{3}$, YAN ZHOU ${ }^{2}$, YANHONG PAN ${ }^{6}$, XIAOMING WEI $^{6}$, \\ SHUANG YANG ${ }^{1}$, FENG MU $^{1}$ and ZHISHENG LIU ${ }^{3}$
}

\begin{abstract}
${ }^{1}$ Department of Research and Development, BGI-Central China, Wuhan East Lake High-Tech Development Zone, Wuhan, Hubei 430075; Departments of ${ }^{2}$ Obstetrics and ${ }^{3}$ Neurology, Wuhan Medical and Health Center for Women and Children, Wuhan, Hubei 430016; ${ }^{4}$ Department of Neurology, Wuhan Children's Hospital, Wuhan, Hubei 430015; ${ }^{5}$ Department of Radiology,

Union Hospital, Tongji Medical College, Huazhong University of Science and Technology, Wuhan, Hubei 430074;

${ }^{6}$ Department of Research and Development, BGI-Shenzhen, Shenzhen, Guangdong 518083, P.R. China
\end{abstract}

Received February 21, 2014; Accepted November 14, 2014

DOI: $10.3892 / \mathrm{mmr} .2014 .3135$

\begin{abstract}
Mutations in the LAMA2 gene cause laminin $\alpha-2$ (merosin)-deficient congenital muscular dystrophies, which are autosomal recessive muscle disorders. Laminin $\alpha-2$ is widely expressed in the basement membrane of skeletal muscle, the myotendinous junctions and extra-synaptically at neuromuscular synapses. In the present study, target next-generation sequencing was used for mutation detection, and polymerase chain reaction (PCR) analysis and Sanger sequencing were used in the identification of small deletions. Subsequently, quantitative PCR (qPCR) was performed to characterize the identified deletion encompassing exon five of the LAMA2 gene. Two causative mutations were identified using target region sequencing which provided the additional information required to facilitate clinical diagnosis. One heterozygous mutation (p. Lys682LysfsX22) was identified and confirmed by Sanger sequencing, and another heterozygous mutation (Exon5del) was found and validated by qPCR. Co-segregation analysis indicated that the Exon5del mutation originated from the proband's mother and the previously reported frameshift mutation (p. Lys682LysfsX22) was inherited from the proband's father. To the best of our knowledge, the present study was the first to report an entire exon five deletion in the LAMA2 gene.
\end{abstract}

Correspondence to: Dr Zhisheng Liu, Department of Neurology, Wuhan Medical and Health Center for Women and Children, 100 Xianggang Road, Wuhan, Hubei 430016, P.R. China

E-mail: liuzsc@126.com

*Contributed equally

Key words: congenital muscular dystrophies, LAMA2 gene, next-generation sequencing, mutations, intragenic deletion

\section{Introduction}

Congenital muscular dystrophies (CMD) are a group of neuromuscular disorders characterized by severe muscle hypotonia at birth or within the first years of life. Symptoms include delayed motor milestones, respiratory and swallowing difficulties as well as generalized muscle weakness (1). The major CMD subtypes include merosin-deficient CMD, integrin-deficient CMD, fukuyama CMD, CMD with rigid spine syndrome, muscle-eye-brain disease, Ullrich CMD and Walker-Warburg syndrome (2). Merosin is absent in approximately half of the patients with classical CMD and frequently in the most severe cases. Therefore, classical CMDs are classified into two groups: Merosin-negative and merosin-positive CMD (3). Merosin-deficient CMD is characterized by the absence of the laminin $\alpha 2$ chain around muscle fibres as well as elevated serum creatine kinase (CK) levels, particularly in the early months of life. The majority of patients with merosin-deficient CMD have normal intelligence but certain individuals may exhibit moderate mental retardation and epilepsy (4-6).

LAMA2-associated muscular dystrophy, which is also known as merosin-deficient CMD type $1 \mathrm{~A}(\mathrm{MDC} 1 \mathrm{~A})$, is inherited in an autosomal recessive manner (7). The prevalence of CMDs was estimated to be between 0.7/100,000 (8) and 2.5/100,000 (9), although the precise prevalence of MDC1A in China has remained unknown. The LAMA2 gene spans $260 \mathrm{~kb}$, encompassing 65 exons and transcribing a messenger RNA transcript of 9.5 kb (NM_000426.3) (7). LAMA2 encodes a $400-\mathrm{kDa}$ protein that is post-translationally cleaved into two subunits (300- and $80-\mathrm{kDa}$ ) associated with disulfide bonds (7). In skeletal muscle, LAMA2 has been suggested to mediate the migration, organization and attachment of cells during embryonic development via interactions with components of the extracellular matrix (10). A potential hypothesis for the mechanism underlying the pathogenesis of LAMA2-associated muscular dystrophy was suggested following studies in zebrafish, in which the sarcolemma was 
destabilized due to cellular damage and subsequent apoptosis (11).

At present, next-generation sequencing (NGS) is becoming more widely recognized as a genetic analysis tool $(12,13)$. Unlike traditional direct sequence analyses, NGS provides thousands of reads at single nucleotide resolution. The high-throughput sequencing data generated by such large-scale parallel sequencing enables more accurate quantification of sequence changes. In order to improve efficiency and reduce costs, it is often necessary to select specific regions of interests and enrich these regions prior to sequencing. Targeted NGS, which is based on chip capturing and large-scale parallel sequencing, may present an efficient strategy to circumvent this problem. At present, targeted NGS technology is frequently used to search for alleles underlying Mendelian disorders (14-17).

The present study aimed to demonstrate the utility of targeted NGS by efficiently identifying novel intragenic deletions of the $L A M A 2$ gene in two patients with CMD.

\section{Materials and methods}

Patients. Study participants provided informed written consent, and the study was approved by the Ethics Committee of the Beijing Genomics Institute at Shenzhen (Shenzen, China). Samples were taken at Wuhan Medical and Health Center for Women and Children in November 2012. DNA was isolated from peripheral blood using QIAamp DNA BloodMiNi kit (Qiagen, Hilden, Germany).

The proband was a six-year-old boy who exhibited limb weakness that resulted in difficulties jumping, running and walking. CMD symptoms were identified at birth, indicated by poor spontaneous movements, a weak cry and feeding difficulties. No intellectual deficiencies were identified. Neurological examination revealed that the proximal end of the lower limb muscle strength was graded III, distal muscle strength was graded level III+ and lower limb muscle strength was graded IV (18). Electromyography (EMG) using Nicolet VikingQuest (Thermo Nicolet, Madison, WI, USA) identified a potential myogenic lesion. Magnetic resonance imaging (MRI) of the brain using a Siemens Tro 3.0T superconductive MRI scanner (Siemens Healthcare, Erlangen, Germany). The regular gradient recalled echo sagittal T1-weighted sequence was imaged under the parameters: TR/TE $=400 / 2.5 \mathrm{~ms}$; matrix, 256x320; excitation number, two; slice thickness, $5 \mathrm{~mm}$; slice gap, $1.5 \mathrm{~mm}$. The T1-weighted axial image was taken under the parameters: $\mathrm{TR} / \mathrm{TE}=200 / 2.5 \mathrm{~ms}$; matrix, 256x320; excitation number, two; slice thickness, $5 \mathrm{~mm}$; slice gap, $2 \mathrm{~mm}$. In addition, axial fluid attenuation inversion recovery sequence (FLAIR) was scanned with TR/TI/TE $=8500 / 2440 / 93 \mathrm{~ms}$; matrix, 464x512; excitation number, two. Axial FLAIR and T1-weighted axial image were corresponding, supplemented by coronal FLAIR scanning, which was performed following an identical protocol apart from the direction of imaging. The results revealed abnormal signaling in bilateral symmetry of the white matter (Fig. 1). Concentrations of CK and CK-muscle/brain (MB) isoenzyme were 491 and $28 \mathrm{U} / 1$, respectively. Concentrations of L-lactate dehydrogenase (LDH-L) and lactate dehydrogenase isoenzyme 1 (LDH-1) were 352 and $79 \mathrm{U} / 1$, respectively. The proband tested negative for metabolic enzymes.

Patient two, four months old at the time of examination, was the younger brother of the proband. Patient two was delivered via cesarean section at full-term without asphyxiating. At the time of birth, the parents of patient two identified limb weakness, weak cry and feeding difficulties. Aged sixty days, patient two was able to raise his head, unstably. It took three months for patient two to exhibit a laughing facial expression. Following rehabilitation treatment for $\sim$ one month, patient two appeared alert and responded normally in the physical examination. Head circumference was $40 \mathrm{~cm}$, limb muscle strength was graded IV and muscular tension was normal. The bilateral patellar reflex was active, although the Achilles tendon reflex was not elicited. The cranial nerve examination was normal. Patient two was able to raise his head when he was pulled up in supine position, but not in prone position. A brain MRI indicated bilateral lateral fissure, and the longitudinal and frontal brain extracellular spaces were wider. Electroencephalography results were normal. The results of EMG analysis indicated that patient two may exhibit myogenic damage. The Gesell test (19) demonstrated that the general developmental level was ten weeks when patient two was 15 weeks of age. Concentrations of CK and CK-MB isoenzyme were 5,354 and $197 \mathrm{U} / 1$, respectively. Concentrations of LDH-L and LDH-1 were 755 and $113 \mathrm{U} / 1$, respectively.

Chip capturing, library construction and next-generation sequencing. Total DNA was fragmented into the range of 200-250 bp using an ultrasonoscope (Covaris S2; Covaris, Inc., Woburn, MA, USA). The DNA library was constructed according to standard Illumina protocols (Illumina, Inc., San Diego, CA, USA) (20). Following adaptor ligation, the library was amplified using four-cycle PCR with PCR primers containing a custom-synthesized barcode sequence $(8 \mathrm{bp})$ as a sample index signature, and a high-fidelity Pfx DNA polymerase (Invitrogen Life Technologies, Calsbad, CA, USA). Reactions were conducted in the GeneAmp PCR system 9700 (Applied Biosystems Life Technologies, Foster City, CA, USA). The first phase was $2 \mathrm{~min}$ at $94^{\circ} \mathrm{C}$, followed by the second phase of $15 \mathrm{sec}$ at $94^{\circ} \mathrm{C}, 30 \mathrm{sec}$ at $62^{\circ} \mathrm{C}$ and $30 \mathrm{sec}$ at $72^{\circ} \mathrm{C}$ for four cycles. The third phase was 5 min at $72^{\circ} \mathrm{C}$, followed by $4^{\circ} \mathrm{C}$. A custom-designed capture array (Roche NimbleGen, Inc., Madison, WI, USA) was used to capture neuromuscular genes. The custom-made array targeted all exons plus the flanking $10 \mathrm{bp}$ of the adjacent introns of the target gene. The final PCR products were pooled and hybridized to the capture array for $72 \mathrm{~h}$. The washing, elution and additional amplification steps were performed according to NimbleGen protocols (Roche NimbleGen, Inc.). The captured samples were subsequently analyzed using the Agilent 2100 Bioanalyzer (Agilent Technologies, Inc., Santa Clara, CA, USA) and the ABI StepOne (Applied Biosystems Life Technologies, Foster City, CA, USA) for DNA fragment length measurement and estimation of enrichment magnitude, respectively $(21,22)$. The captured libraries were sequenced on the Illumina genome analyzer (Illumina HiSeq2000 Analyzer) as 90-bp paired-end reads, according to the manufacturer's instructions. Image analysis and base calling were performed using Illumina Pipeline (version 1,3,4). 
A

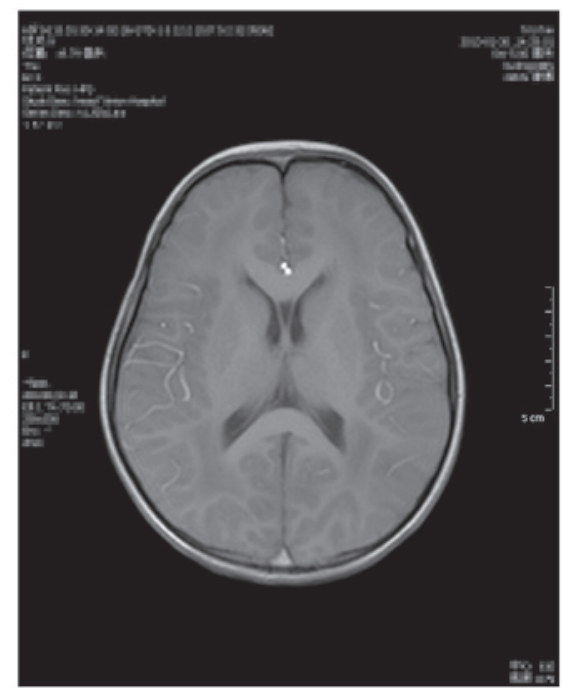

C

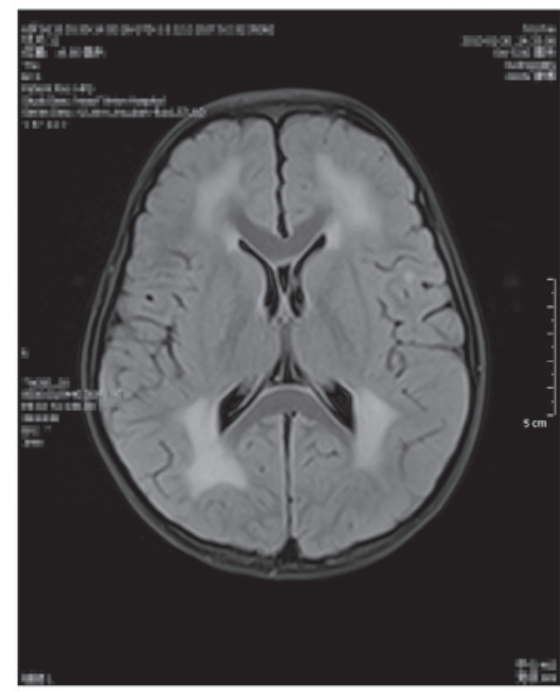

$\mathbf{E}$

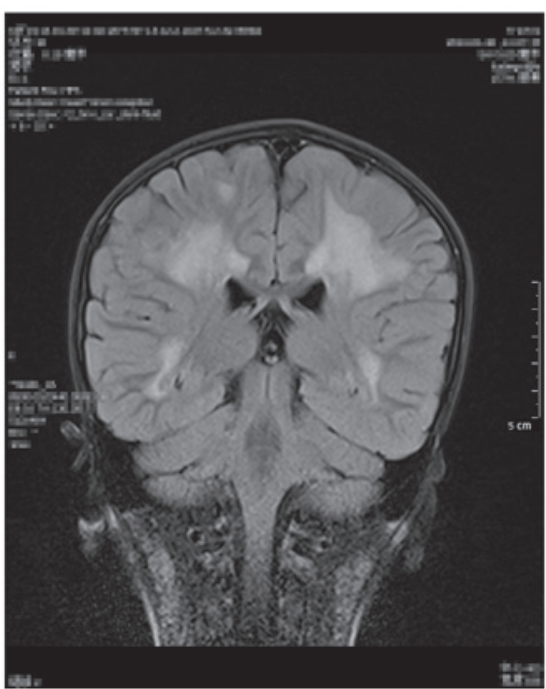

B

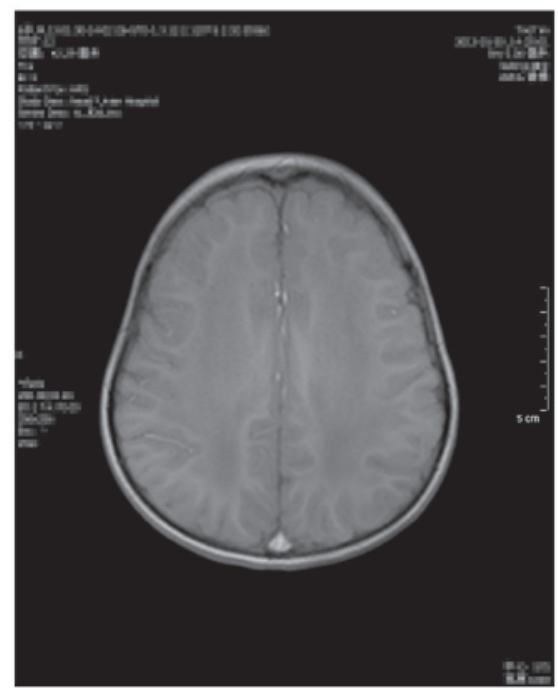

D

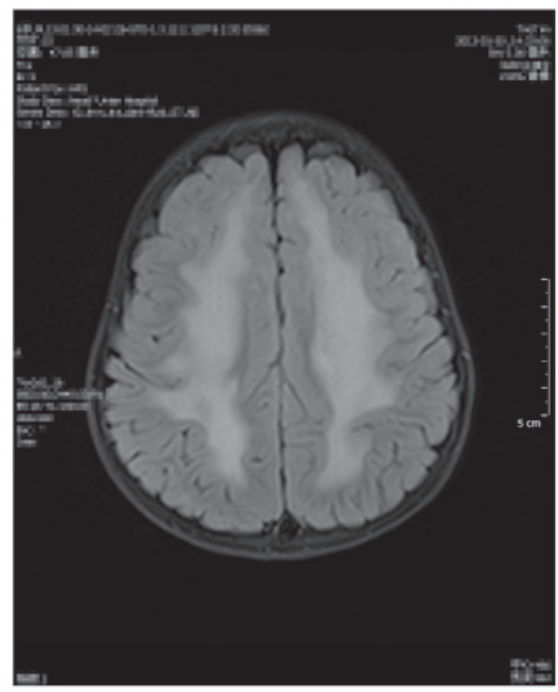

F

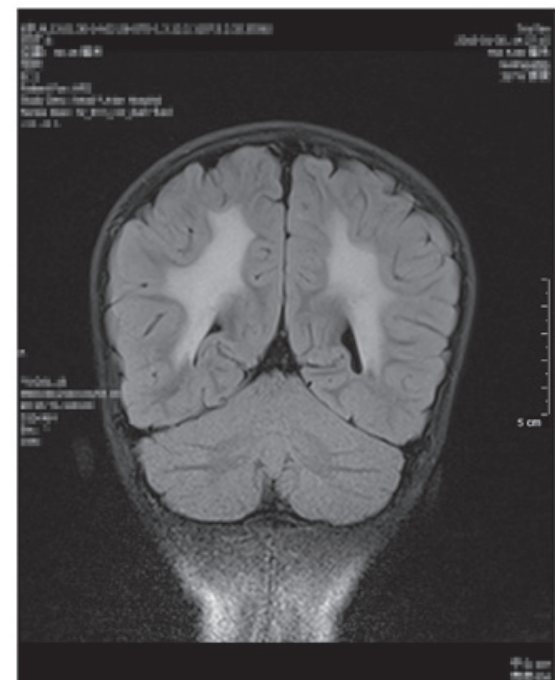

Figure 1. Brain magnetic resonance images of the proband and a healthy control. Axial T1W1 images of (A) the proband and (B) a control brain. Axial T1Wl and FLAIR images of (C) the proband and (D) a control brain. Coronal T1Wl and FLAIR images of (E) the proband and (F) a control brain. FLAIR, fluid-attenuated inversion recovery.

Data filtering and analysis pipeline. Following completion of the entire run, filter criteria were used to remove unqualified sequences from the primary data. Unqualified sequences were defined as reads that contained $>10 \%$ no signal detected in the read length, adapter sequences including indexed sequence and $50 \%$ reads with a quality value of $<5$ as well as an average quality $<10$ (23). Qualified sequences were designated as clean reads for further analysis. Clean reads with a length 
of $90 \mathrm{bp}$ were aligned against the reference human genome (hg19) using the Burrows Wheeler Aligner Multi-Vision software package (http://bio-bwa.sourceforge.net/) (24). The single-nucleotide variants (SNVs) and insertions and deletions (indels) were identified using SOAPsnp software (25) and the SAMtools (26), respectively. Identified SNVs were annotated using four databases, including the Single Nucleotide Polymorphism database (www.ncbi.nlm.nih.gov/projects/ SNP/), the Hapmap project (hapmap.ncbi.nlm.nih.gov/), the 1000 Genomes Project (www.1000genomes.org/) and the 124 healthy reference samples sequenced. These healthy reference samples were obtained from members of staff at BGI-Central China who passed a health screen. Known disease-causing mutations were identified from the Leiden Open Variation Database (www.lovd.nl/).

Sanger sequencing. PCR products containing potential variants were analyzed using Sanger sequencing in order to ascertain the accuracy of variant identification by NGS The specific primers were designed by Primer 6.0 software (Premier Biosoft, Palo Alto, CA, USA). The Sanger sequencing was performed using a 3730xl DNA Analyzer (Applied Biosystems Life Technologies) according to a previously described protocol (23).

Copy number of exons in LAMA2. To determine the copy number $(\mathrm{CN})$ of exons in the gene in a given sample, intraand inter-sample normalization were performed. For each sample subjected to intra-sample analysis, the relative depth count of an exon was defined as the ratio of the depth count of an exon over the total depth count of exons in the gene to which the exons belong. Inter-sample normalization was subsequently performed to divide the relative depth count of each exon by the corresponding relative depth count of the control sample in the same experiment. In short: $\mathrm{CN}=\left(\mathrm{depth}^{\mathrm{i}} / \mathrm{depth}^{\text {all }}\right)_{\text {test }} /\left(\mathrm{depth}^{\mathrm{c}-\mathrm{i}} / \mathrm{depth}^{\mathrm{c}-\mathrm{all}}\right)_{\text {ref }}$, where depth $^{\mathrm{i}}=$ depth of $\mathrm{i}$ exon; depth ${ }^{\text {all }}=$ depth of all exons in one gene; depth $^{\mathrm{c}-\mathrm{i}}=$ depth of i exon in control sample; depth ${ }^{\mathrm{c}-\mathrm{all}}=$ depth of all exons in one gene in control sample; test=test individual and ref=reference individual $(27,28)$.

qPCR analysis. To further quantify the intragenic deletions in the specimens from patients with CMD, qPCR with SYBR Green (Takara Bio, Inc., Otsu, Japan) was performed in the StepOne $^{\mathrm{TM}}$ Real-Time PCR System (Applied Biosystems Life Technologies) according to the manufacturer's instructions. All primers were designed using Primer 6.0 and tested for specificity using National Center for Biotechnology Information Basic Local Alignment Search Tool software (blast.ncbi.nlm. nih.gov/Blast.cgi). The housekeeping gene, GAPDH, was used as an endogenous control. Measurements were repeated $\geq$ three times to verify the reproducibility of the results. The cycling conditions were as follows: $10 \mathrm{~min}$ at $95^{\circ} \mathrm{C}, 40$ cycles at $95^{\circ} \mathrm{C}$ for $15 \mathrm{sec}$ and $60^{\circ} \mathrm{C}$ for $30 \mathrm{sec}$. Following PCR amplification, a melting curve was generated for each PCR product to confirm reaction specificity.

Statistical analysis. Statistical analyses were performed using Microsoft Office Excel 2007 (Albuquerque, NM, USA). Values are expressed as the mean \pm standard error.
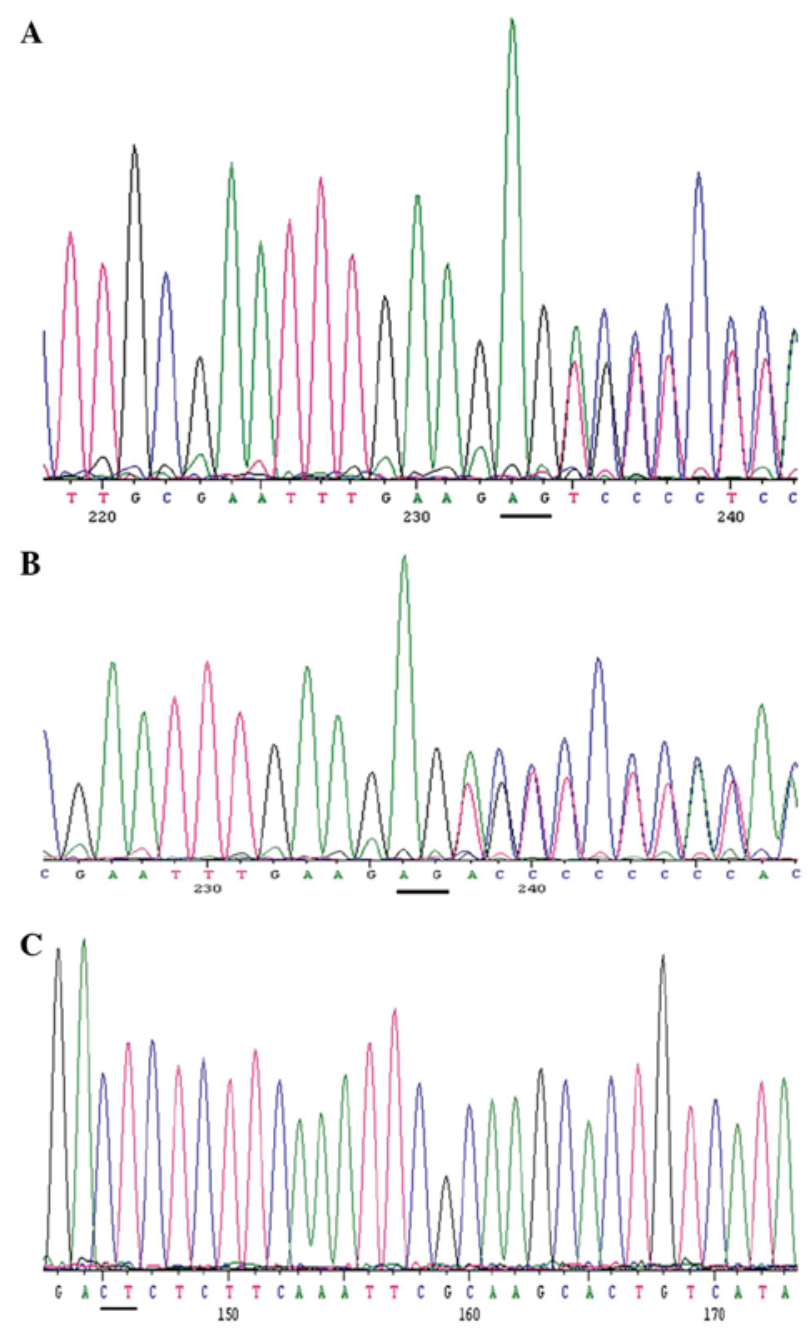

Figure 2. Co-segregation analysis of the proband and family. (A) Short, heterozygous deletion of two base pairs verified in the DNA of the proband's father. (B) A heterozygous deletion of two base pairs was confirmed in the DNA of the proband. (C) The DNA sequence of the proband's mother was reverse and normal. The loci are indicated by a black, horizontal line.

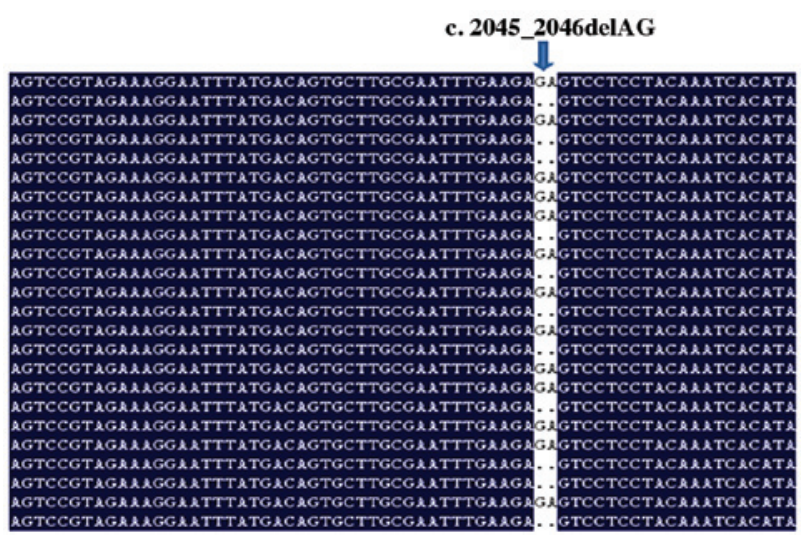

Figure 3. A small number of reads spanning the mutation site of the proband were expanded to reveal the deleted DNA sequence in certain mapped reads. Arrow, changed base.

\section{Results}

Identification of candidate mutations in the LAMA2 gene. 


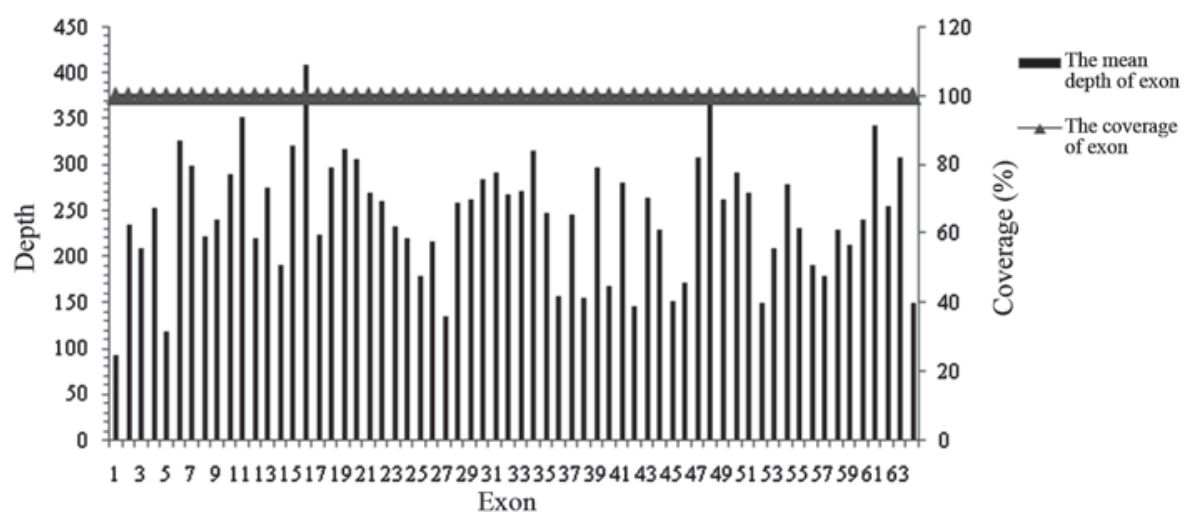

Figure 4. Average depth plot of the proband. Each bar indicates the average sequencing depth, and the line demonstrates the coverage for 64 exons in the LAMA2 gene of the proband.

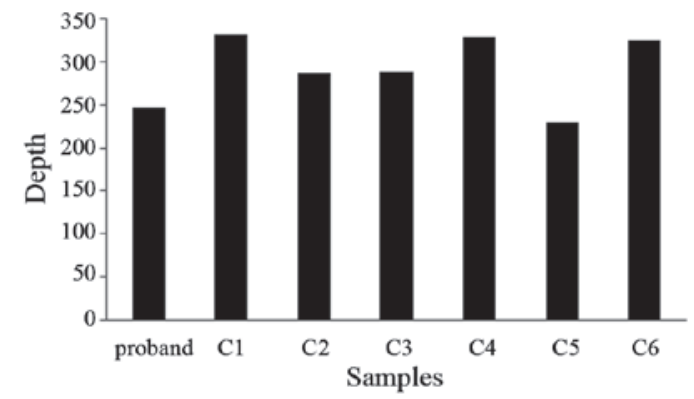

Figure 5. Average sequencing depth for all exons of LAMA2 in all of the samples tested. The average sequencing depth was $>227$-fold in each sample.

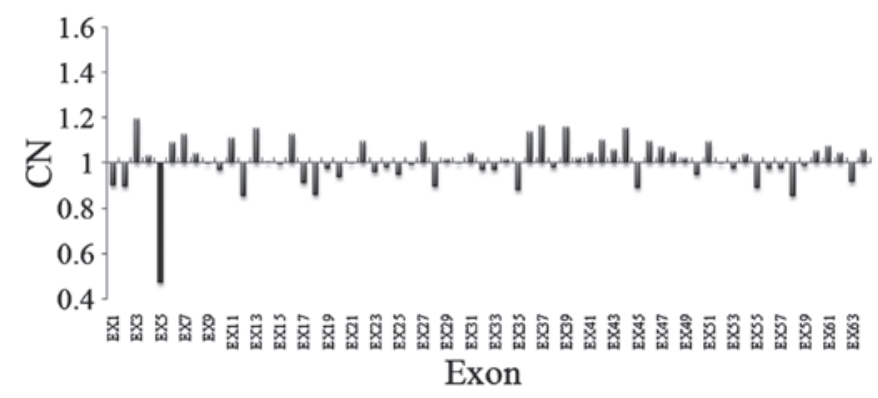

Figure 6. CN plot. Each bar indicates the relative copy number of each exon of the proband. The average relative depth count of six normal samples was used as a reference. $\mathrm{CN}$, copy number; Ex, exon.

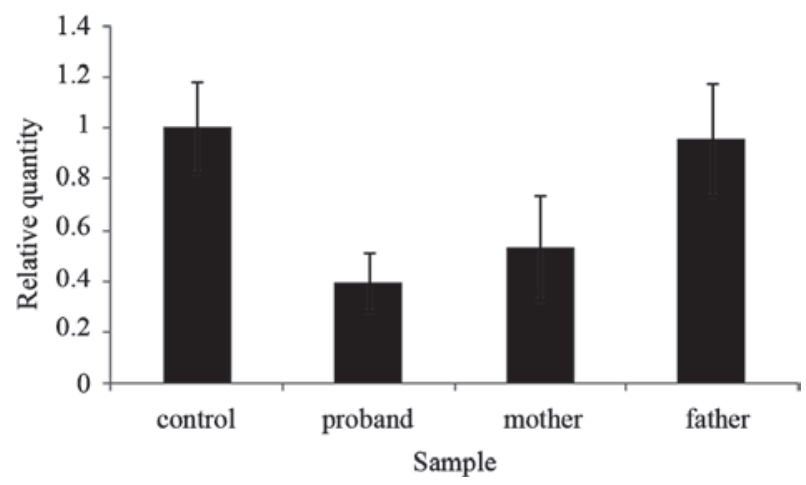

Figure 7. Verification of the deletion mutation in the family of the proband using real-time polymerase chain reaction analysis. The relative quantity of exon five in the proband and the proband's mother was almost half of that of the proband's father and the control. Values are expressed as the mean \pm standard error.
To identify the most probable pathogenic mutations, all SNVs previously reported in the four databases described were excluded. The common variants (indel and substitution) were filtered out if their frequency was $>0.05$ in any of the four databases. Only mutations in the coding DNA sequence regions were selected. One compound heterozygous mutation was identified in the LAMA2 gene in the proband (c. 2045_2046delAG). A pair of primers was designed for each specific site to test four family members, including the proband and patient two (data not shown), LAMA-F-1 (5'-AGTCTATTGAGGGTGGAGGATACA-3') and LAMA-R-1 (5'-GGCTCCGTTCTTATCTGCTCTT-3'), and the PCR products were validated by Sanger sequencing. A small deletion in LAMA2 was confirmed in the proband (Fig. 2A). Concurrently, the potential site was validated by PCR and Sanger sequencing in the proband's father (Fig. 2B), but not in the mother (Fig. 2C). The (c. 2045_2046delAG) mutation causes a frameshift and introduces a premature stop codon that may shorten the protein transcribed (29). Read mapping was used to analyze the two base pair deletion in the proband (Fig. 3).

Identification of a deletion of exon five of LAMA2 (Exon5del) in the proband. In the present study, the average sequencing depth of all exons of the LAMA2 gene were analyzed in the proband and controls using targeted NGS. The mean sequencing depth of the target region was $>227$-fold in each sample and on average, $100 \%$ of the targeted region was covered in each sample, indicating that the method was reliable at detecting DNA variants (Figs. 4 and 5). In addition to specific and accurate variant detection, simultaneous $\mathrm{CN}$ variation analysis within the same targeted NGS experiment was significant for diagnostic purposes. The average relative depth count of six normal samples was used as a reference. Each mean depth of exons of LAMA2 from the proband was divided by the corresponding average depth of exons from the reference sample. Based on the observations presented in Fig. 6, exon five was selected for subsequent screening.

The Exon5del mutation is on the maternal allele. To confirm the deletion of exon five of the LAMA2 gene in the proband, an exon quantification strategy based on PCR was utilized. qPCR was used to detect the DNA copy number of the exons in the 
proband, the proband's mother and father, patient two (data not shown) and a normal control, where the genomic GAPDH gene was used as a loading control. The primers were as follows: Forward, 5'-GACCAAGTGCCGATGATCCTTC-3' and reverse, 5'-GTCAGCATTCAGTGTGCGGAT-3'. The results indicated that the quantity of exon five detected in the DNA sample of the proband was $\sim 50 \%$ of that of his father and the control samples, and almost $100 \%$ of that of his mother's sample (Fig. 7). These results suggested that there was a heterozygous deletion of exon five in the LAMA2 gene in the proband and the proband's mother (30). It was therefore concluded that the deletion of LAMA2 exon five occurred on the maternal allele, and that this heterozygous mutation was inherited by the proband.

\section{Discussion}

The comprehensive study of single-gene disorders and the corresponding advances in technologies available for disease detection has meant that increasing numbers of monogenic inherited diseases may be identified by clinical molecular diagnosis (31). There are numerous drawbacks of routine molecular diagnosis, including genetic and clinical disease heterogeneity, unidentified genes, non-specific clinical features and the involvement of large and/or multiple genes requiring complementary methods, which result in an increased diagnosis time and delays in the subsequent molecular validation of the diagnosis (32). Second generation sequencing technology and directional capture tools have facilitated the development of novel clinical molecular diagnostic techniques to detect single gene disorders (33). The advantage of this approach is that the techniques are relatively low-cost, produce data that are easier to interpret and allow corrected diagnosis, which confirms the genetic heterogeneity of diseases (34).

The clinical manifestations observed in the proband and patient two suggested that they may have had congenital muscular dystrophy. The disease was subsequently diagnosed and classified accurately via genetic testing, as indicated by the results of the present study. Using a combination of targeted-gene enrichment and NGS technology, an intragenic deletion on the maternal allele and a two base pair deletion on the paternal allele were identified in the proband. To confirm the candidate variants, genomic qPCR and Sanger sequencing were used. If a conventional sequencing method had been used it would have been unlikely to detect the large intragenic $L A M A 2$ gene deletion identified in the present study.

In LAMA2, which comprises 64 exons, mutations are widely distributed and there is no clear hot-spot amongst identified mutations (35-37). The small deletion (p. Lys682LysfsX22) potentially causes a frame shift, resulting in premature translational termination at amino acid 682 - a mutation previously confirmed to be disease-causing (29) - yielding a non-functional protein product. The entire deletion of exon five was a novel mutation identified in a CMD patient in the present study. The protein containing the intragenic deletion mutation was damaged as exon five comprised part of domain IV of laminin $\alpha-2$. Domain IV participates in calcium ion-dependent intermolecular interactions and integrin binding (38), and is highly conserved in the laminin isoforms of numerous human and non-human species. The two identified mutations were additionally confirmed in patient two (data not shown). Genetic testing has an increasingly significant role in the diagnosis of patients with elusive muscular dystrophy phenotypes, particularly in the absence of known family history.

In conclusion, the present study indicated that an integrated approach, combining exon-capture sequencing and clinical molecular diagnosis, was a fast, efficient and a reliable diagnostic tool for congenital myopathies. To the best of our knowledge, the present study was the first to report the deletion of exon five in $L A M A 2$ in a Chinese family.

\section{Acknowledgements}

The authors would like to thank the blood donors for their contribution to the present study. The present study received financial support from the National High-Tech Research and Development Program of China (863 Program; no. 2012AA02A201), the Guangdong Enterprise Key Laboratory of Human Disease Genomics (Shenzen, China), Shenzhen Key Laboratory of Transomics Biotechnologies (Shenzen, China; no. CXB201108250096A), Shenzhen Engineering Laboratory for Clinical Molecular Diagnostics (Shenzen, China) and the China National GeneBank (Shenzen, China).

\section{References}

1. Allamand V and Guicheney P: Merosin-deficient congenital muscular dystrophy, autosomal recessive (MDC1A. MIM\#156225,LAMA2 gene coding for alpha2 chain of laminin). Eur J Hum Genet 10: 91-94, 2002.

2. Sparks S, Quijano-Roy S, Harper A, et al: Congenital Muscular Dystrophy Overview, 2012. In: GeneReviews ${ }^{\circledR}$ [Internet]. Pagon RA, Bird TD, Dolan CR, et al (eds). University of Washington, Seattle, WA, 1993-2014.

3. Kobayashi O, Hayashi Y, Arahata K, Ozawa E and Nonaka I: Congenital muscular dystrophy: Clinical and pathologic study of 50 patients with the classical (Occidental) merosin-positive form. Neurology 46: 815-818, 1996.

4. Louhichi N, Triki C, Quijano-Roy S, Richard P, Makri S, Méziou M, Estournet B, Mrad S, Romero NB, Ayadi H, et al: New FKRP mutations causing congenital muscular dystrophy associated with mental retardation and central nervous system abnormalities. Identification of a founder mutation in Tunisian families. Neurogenetics 5: 27-34, 2004.

5. Voit T, Cirak S, Abraham S, Karakesisoglou I, Parano E, Pavone P, Falsaperla R, Amthor H, Schroeder J, Mutoni F, et al: Congenital muscular dystrophy with adducted thumbs, mental retardation, cerebellar hypoplasia and cataracts is caused by mutation of Enaptin (Nesprin-1): The third nuclear envelopathy with muscular dystrophy. In: Proceedings of the 12th International Congress of the World-Muscle-Society Italy. Neuromuscular Disorders, Pergamon-Elsevier Science Ltd, Oxford, UK, pp833-834, 2007.

6. Messina S, Tortorella G, Concolino D, Spanò M, D'Amico A, Bruno C, Santorelli FM, Mercuri E and Bertini E: Congenital muscular dystrophy with defective alpha-dystroglycan, cerebellar hypoplasia, and epilepsy. Neurology 73: 1599-601, 2009.

7. Quijano-Roy S, Sparks S and Rutkowski A: LAMA2-Related Muscular Dystrophy, 2012. In: GeneReviews ${ }^{\circledR}$ [Internet]. Pagon RA, Bird TD, Dolan CR, et al (eds), University of Washington, Seattle, WA, 1993-2014.

8. Mostacciuolo ML, Miorin M, Martinello F, Angelini C, Perini P and Trevisan CP: Genetic epidemiology of congenital muscular dystrophy in a sample from north east Italy. Hum Genet 97: 277-279, 1996.

9. Darin N and Tulinius M: Neuromuscular disorders in childhood: a descriptive epidemiological study from western Sweden. Neuromuscul Disord 10: 1-9, 2000.

10. Suzuki N, Yokoyama F and Nomizu M: Functional sites in the laminin alpha chains. Connect Tissue Res 46: 142-152, 2005. 
11. Hall TE, Bryson-Richardson RJ, Berger S, Jacoby AS, Cole NJ, Hollway GE, Berger J and Currie PD: The zebrafish candyfloss mutant implicates extracellular matrix adhesion failure in laminin alpha2-deficient congenital muscular dystrophy. Proc Natl Acad Sci USA 104: 7092-7097, 2007.

12. Yang Y, Muzny DM, Reid JG, et al: Clinical whole-exome sequencing for the diagnosis of mendelian disorders. N Engl J Med 369: 1502-1511, 2013.

13. Biesecker LG, Burke W, Kohane I, et al: Next-generation sequencing in the clinic: are we ready? Nat Rev Genet 13: 818-824, 2012.

14. Iqbal Z, Neveling K, Razzaq A, Shahzad M, Zahoor MY, Qasim M, Gilissen C, Wieskamp N, Kwint MP, Gijsen S, et al: Targeted next generation sequencing reveals a novel intragenic deletion of the TPO gene in a family with intellectual disability. Arch Med Res 43: 312-316, 2012.

15. Wei X, Jin F, Ye Y, Xu C, Qu N, Ju X and Yi X: A novel mutation of IDS gene in a Chinese patient with mucopolysaccharidosis II by next-generation sequencing. Clin Chim Acta 412: 2340-2342, 2011.

16. Wei X, Sun Y, Xie J, Shi Q, Qu N, Yang G, Cai J, Yang Y, Liang Y, Wang W and Yi X: Next-generation sequencing identifies a novel compound heterozygous mutation in MYO7A in a Chinese patient with Usher Syndrome 1B. Clin Chim Acta 413: 1866-1871, 2012.

17. Jones MA, Bhide S, Chin E, Ng BG, Rhodenizer D, Zhang VW, Sun JJ, Tanner A, Freeze HH and Hegde MR: Targeted polymerase chain reaction-based enrichment and next generation sequencing for diagnostic testing of congenital disorders of glycosylation. Genet Med 13: 921-932, 2011.

18. Mendell JR and Florence J: Manual muscle testing. Muscle Nerve 13 (Suppl): S16-S20, 1990.

19. Ames LB, Gillespie BS, Haines J and Ilg FL: The Gesell Institute's child from one to six: Evaluating the behavior of the preschool child. Harper \& Row, New York, 1979.

20. Illumina Protocol for Whole Genome Sequencing using SBS Technology. BioTechniques Protocol Guide. Biotechniques, New York, NY, 2006.

21. Chilamakuri CS, Lorenz S, Madoui MA, Vodák D, Sun J, Hovig E, Myklebost O and Meza-Zepeda LA: Performance comparison of four exome capture systems for deep sequencing. BMC Genomics 15: 449, 2014.

22. Guo G, Sun X, Chen C, Wu S, et al: Whole-genome and whole-exome sequencing of bladder cancer identifies frequent alterations in genes involved in sister chromatid cohesion and segregation. Nat Genet 45: 1459-1463, 2013.

23. Wei X, Ju X, Yi X, Zhu Q, Qu N, et al: Identification of sequence variants in genetic disease-causing genes using targeted next-generation sequencing. PLoS One 6: e29500, 2011.

24. Li H and Durbin R: Fast and accurate long-read alignment with Burrows-Wheeler transform. Bioinformatics 26: 589-595, 2010.
25. Li R, Li Y, Fang X, Yang H, Wang J, Kristiansen K and Wang J: SNP detection for massively parallel whole-genome resequencing. Genome Res 19: 1124-1132, 2009.

26. Li H, Handsaker B, Wysoker A, Fennell T, Ruan J, Homer N, Marth G, Abecasis G and Durbin R; 1000 Genome Project Data Processing Subgroup: The Sequence alignment/map (SAM) format and SAMtools. Bioinformatics 25: 2078-2079, 2009.

27. Goossens D, Moens LN, Nelis E, Lenaerts AS, Glassee W, Kalbe A, Frey B, Kopal G, De Jonghe P, De Rijk P and Del-Favero J: Simultaneous mutation and copy number variation $(\mathrm{CNV})$ detection by multiplex PCR-based GS-FLX sequencing. Hum Mutat 30: 472-476, 2009.

28. Kumps C, Van Roy N, Heyrman L, Goossens D, Del-Favero J, Noguera R, Vandesompele J, Speleman F and De Preter K: Multiplex Amplicon Quantification (MAQ), a fast and efficient method for the simultaneous detection of copy number alterations in neuroblastoma. BMC Genomics 11: 298, 2010.

29. Guicheney P, Vignier N, Zhang X, He Y, Cruaud C, Frey V, Helbling-Leclerc A, Richard P, Estournet B, Merlini L, et al: PCR based mutation screening of the laminin alpha 2 chain gene (LAMA2): application to prenatal diagnosis and search for founder effects in congenital muscular dystrophy. J Med Genet 35: 211-217, 1998

30. Hellemans J, Mortier G, De Paepe A, Speleman F and Vandesompele $\mathrm{J}$ : qBase relative quantification framework and software for management and automated analysis of real-time quantitative PCR data. Genome Biol 8: R19, 2007.

31. Katsanis SH and Katsanis N: Molecular genetic testing and the future of clinical genomics. Nat Rev Genet 14: 415-426, 2013.

32. Vasli $\mathrm{N}$ and Laporte $\mathrm{J}$ : Impacts of massively parallel sequencing for genetic diagnosis of neuromuscular disorders. Acta Neuropathol 125: 173-185, 2013.

33. Bell CJ, Dinwiddie DL, Miller NA, et al: Carrier testing for severe childhood recessive diseases by next-generation sequencing. Sci Transl Med 3: 65ra4, 2011.

34. Umbarger MA, Kennedy CJ, Kennedy CJ, Saunders P, et al: Next-generation carrier screening. Genet Med 16, 132-140, 2014.

35. Oliveira J, Santos R, Soares-Silva I, Jorge P, et al: LAMA2 gene analysis in a cohort of 26 congenital muscular dystrophy patients. Clin Genet 74: 502-512, 2008.

36. Di Blasi C, Piga D, Brioschi P, et al: LAMA2 gene analysis in congenital muscular dystrophy: new mutations, prenatal diagnosis, and founder effect. Arch Neurol 62: 1582-1586, 2005.

37. Yamamoto LU, Gollop TR, Naccache NF, et al: Protein and DNA analysis for the prenatal diagnosis of alpha2-laminin-deficient congenital muscular dystrophy. Diagn Mol Pathol 13: 167-171, 2004.

38. Engvall E and Wewer UM: Domains of laminin. J Cell Biochem 61: 493-501, 1996. 